\title{
Tratamiento de úlcera por presión grado IV con cierre asistido por vacío
}

\author{
Vaccum-Assisted IV-Degree Pressure \\ Sore Treatment
}

Lic. Enf. Imelda Flores Montes*, Lic. Enf. Claudia Leija Hernández**

*Clínica de Manejo Avanzado de Heridas, **Jefe del Departamento de Administración General del Instituto Nacional de Cardiología Ignacio Chávez.

\section{Resumen}

Las úlceras por presión son lesiones producidas por la tensión que se sufre al permanecer largos periódos sobre la piel en una sola posición, este tipo de heridas representa un importante problema asistencial por el impacto sobre la salud y la calidad de vida. La prevención y tratamiento de estas úlceras involucra al personal de enfermería, médicos y cirujanos, así como familiares y principalmente al paciente para su cooperación durante su recuperación. Actualmente, para el tratamiento de las úlceras grado III y IV se utiliza el cierre asistido por vacío el cual es un sistema que favorece la cicatrización de las heridas.

Este artículo describe el caso de un paciente que presenta una úlcera por presión Grado IV, permanece con sistema

The ulcers by pressure are injuries produced by tension on the skin remaining long periods in a single position; this wounds represents an important problem by the impact on the health and the quality of life. The prevention and treatment of these ulcers involve nurses, doctors and surgeons, family and the patient's cooperation during its recovery. At the moment, for the treatment of the ulcers degree III and IV the Vacuum Assisted Closure (VAC) is used like a system that favors the healing of the wounds. This article describes the case of a patient who had an ulcer by pressure degree IV, with system VAC, during 12 weeks
VAC durante 12 semanas hasta la programación para cierre quirúrgico, el cual se decide al encontrarse en condiciones clínicas favorables. La terapia asistida por vacío ha mostrado su eficacia permitiendo acelerar el proceso de formación de tejido de granulación y disminuir en forma importante el tamaño de la lesión, además de convertirse en una herida cerrada y controlada evita la presencia de infección, permitió egresar tempranamente al paciente ya que se utilizó un sistema portátil con lo que se logró que regresara a sus actividades cotidianas.

Palabras clave: Úlcera por presión, cierre asistido por vacío, colgajo miocutáneo.

\section{Abstract}

until before that the surgical closing, when he was in favorable clinical conditions. The VAC show its effectiveness accelerating the process of granulation, with important diminish the size of the injury, besides to close the wound, its control and avoid infection, allowed to withdraw early to the patient since a portable system was used with which it was obtained that it returned to his daily activities.

Key words: Ulcer by pressure, Vacuum Assisted Closure, Myocutaneous Flap.

\section{INTRODUCCIÓN}

Podemos definir una úlcera por presión (UPP) como toda pérdida tisular, producida por isquemia y derivada de una presión ejercida y mantenida sobre una prominencia ósea, a pesar de que se utilizan de forma intercambiable diversos términos para describir esta pérdida tisular, como úlcera por el lecho o úlcera por decúbito, el término más adecuado es el de úlcera por presión ya que refleja el concepto actual sobre su etiología: una presión sostenida y mantenida sobre la piel por encima del limite tolerable de la misma'. 
Las úlceras por presión representan un importante problema asistencial dada su prevalencia y el impacto sobre la salud y calidad de vida de los pacientes, al elevar el riesgo de infecciones y complicaciones que pueden prolongar la estancia hospitalaria con el consecuente aumento de los costos; todo lo anterior puede influir en la percepción del paciente de que la atención de enfermería es deficiente. La OMS utiliza "la incidencia de las úlceras por presión" como uno de los indicadores para evaluar la calidad asistencial en un país².

Su tratamiento y recuperación depende del grado, localización y extensión de la úlcera, probándose que las úlceras grado III sanan en uno a tres meses y la úlcera grado IV en meses o años y en algunos casos no consiguen su curación total ${ }^{3}$. Para el tratamiento de este tipo de lesiones se ha estado utilizando la curación avanzada, que es la que se realiza en un ambiente húmedo fisiológico, con apósitos activos y la frecuencia de cambio depende de las condiciones locales de la lesión ${ }^{4}$, actualmente se recomienda el uso del sistema V.A.C. (cierre asistido por vacío) el cual es un sistema no invasivo, que favorece la cicatrización, aplicando presión negativa (subatmosférica) en la herida, para descomprimir un apósito de espuma de forma continua o intermitente, además proporciona un entorno húmedo y cerrado para la cicatrización, reduce el volumen de la herida y favorece la eliminación del exudado 5 .

Para la aplicación del tratamiento se deben conocer las bases teóricas que permitan la toma de decisiones como es la fisiopatología, de esta manera se desarrolla un plan de cuidados en base a las necesidades individualizadas para organizar un método terapéutico y asegurar el éxito del mismo.

El presente artículo describe el caso de un paciente con úlcera por presión tratado con sistema VAC para preparación del lecho de la herida y de esta manera recibir colgajo miocutáneo, con resultado exitoso.

\section{MARCO TEORICO}

\section{Fisiopatología de la úlcera por presión}

\section{Concepto:}

Lesión de origen isquémico localizado en la piel y tejidos subyacentes, producida por la acción combinada de factores extrínsecos, entre los que destacan las fuerzas de presión, tracción y fricción; siendo determinante la relación tiempo presión.

- Etiología:

- Presión
- Fricción

- Cizallamiento o fuerza externa de pinzamiento vascular

- Maceración de la piel

\section{Clasificación:}

GRADO I. Limitada a la capa superficial de la piel, se manifiesta a través de enrojecimiento, se mantiene la integridad de la piel.

GRADO II. Afecta a las capas superficiales de la piel y se prolonga a la primera porción del tejido graso subyacente, se manifiesta con erosiones, ampollas y/o desgarros superficiales.

GRADO III. Se extiende más profundamente a través de estructuras superficiales, afecta a la grasa subcutánea, produciendo necrosis del tejido comprometido y alcanza, pero sin abarcar, los músculos.

GRADO IV. Destrucción de todo el tejido blando desde la piel hasta el hueso. Tratamiento:

- Eliminar o aliviar la causa.

- Prevención de nuevas lesiones.

- Soporte nutricional.

- Soporte emocional.

- Control del dolor.

- Cuidado de la piel periúlceral.

- Aplicación de Terapia Húmeda.

- Cierre Asistido por vacío. Reparación quirúrgica. ${ }^{6}$

\section{METODOLOGÍA Descripción del caso}

Se efectúa una valoración inicial que consiste en la evaluación del paciente en forma integral, profundizando sobre las condiciones de la herida para determinar tratamiento. Se obtienen los siguientes datos:

- Identificación del paciente: RMB

- Sexo: Masculino

- Edad: 50 años

- Estado nutricional: Disminución de peso20 kgs.

- Antecedentes: Choque cardiogénico por infarto agudo al miocardio, proceso infeccioso respiratorio durante su estancia de un mes en otro Hospital

- Diagnóstico: Síndrome febril en estudio

- Estado general: Delicado

- Estudios de laboratorio:

- Leucocitos 7.7, plaquetas 366,

- Proteínas 6.0, Albúmina 3.2, Hb 9.4.

- Fecha de ingreso al Instituto: 5-12-06 
A su ingreso se evalúa el estado de la lesión para determinar tratamiento, observando los siguientes aspectos:

- Localización: Sacro

- Fecha de aparición: Agosto 2006

- Fecha de identificación: 6-12-2006

- Estadio: IV

- Tamaño: $8 \times 5 \mathrm{~cm}$

- Lecho de la herida: Necrótico

- Borde de la Herida: Necrótico

- Piel periúlceral: Eritematosa

- Exudado: Nulo

- Signos de infección: Borde con eritema, fiebre de $38^{\circ} \mathrm{C}$.

- Dolor: Nulo

Se selecciona tipo de tratamiento: Sistema de cierre asistido por vacío

Se documenta con registro fotográfico al inicio y después de cada curación. Además se toman las dimensiones de la herida utilizando pinzas para calcular la profundidad y las cavitaciones desde el borde de la piel hasta el contacto con el fondo.

Se realizan curaciones de dos a tres veces por semana por parte de la clínica de heridas.

\section{VALORACIÓN}

Paciente masculino (RMB) de 50 años de edad con diagnóstico médico de Cardiopatía isquémica, antecedentes de internamiento previo en hospital privado por choque cardiogénico durante 1 mes. Desde su egreso presenta úlcera por presión en región sacra cubierta con "apósito". Ingresa al Instituto Nacional de Cardiología Ignacio Chávez por Síndrome febril en estudio, al $1^{\text {er }}$ día de su estancia hospitalaria se reporta úlcera en región sacra cubierta con tejido necrótico por lo que se decide eliminar la placa, quedando úlcera con 95\% de tejido esfacelado, borde necrótico, exudado escaso, con eritema perilesional y sin referir dolor a la manipulación, se deja terapia húmeda durante 24 horas para posteriormente colocar sistema V.A.C.

\section{PLAN DE ATENCIÓN DE ENFERMERÍA Necesidad: Oxigenación Diagnóstico de enfermería 1}

Alto riesgo de deterioro del Intercambio gaseoso relacionado con el desequilibrio entre la ventilación perfusión.

\section{Objetivo}

Continuar con un intercambio gaseoso adecuado

\section{Intervenciones}

Fomento de actividad física, según su capacidad funcional.
Explicar signos de alarma de evento isquémico.

Registrar y valorar signos vitales.

Monitorizar saturación de oxigeno durante su estancia hospitalaria.

\section{Evaluación}

El paciente no presenta deterioro en el intercambio gaseoso durante el tratamiento.

Necesidad: Nutrición

\section{Diagnóstico de enfermería 2}

Desequilibrio nutricional por defecto relacionado con incapacidad para absorber los nutrientes manifestado por peso corporal inferior.

\section{Objetivo}

Mantener el peso ideal durante el tratamiento hasta la resolución de la úlcera.

\section{Intervenciones}

Educación para la salud en cuanto a nutrición.

Fomentar la alimentación adecuada.

Monitorizar con exámenes de laboratorio (proteínas y albúmina).

Registro y valoración de peso y talla.

\section{Evaluación}

El paciente aumento su peso corporal (10\%) en 8 semanas. Necesidad: Movilización

\section{Diagnóstico de enfermería 3}

Deterioro de la movilidad física relacionado con restricciones impuestas al movimiento (por aparataje) manifestado por limitación de la capacidad para habilidades motoras acostumbradas.

\section{Objetivo}

Fomentar la deambulación y la realización de actividades cotidianas.

\section{Intervenciones}

Elegir aparato de succión continua portátil.

Minimizar los cambios en la curación.

Egresar a domicilio en el menor tiempo posible.

\section{Evaluación}

El paciente egresa a domicilio a la primera semana de inicio de tratamiento y se da seguimiento por la consulta externa.

Necesidad: Reposo / Sueño

\section{Diagnóstico de enfermería 4}

Deterioro del patrón de sueño relacionado con ruido causado por la terapia de succión continua manifestado por tiempo de sueño durante el procedimiento. 


\section{Objetivo}

Favorecer el descanso óptimo durante la noche.

\section{Intervenciones}

Alternar tratamiento de succión continua con terapia húmeda.

Fijar apósito adhesivo con el fin de evitar fugas.

\section{Evaluación}

El paciente refiere descanso favorable durante la noche.

Necesidad: Temperatura

\section{Diagnóstico de enfermería 5}

Alto riesgo de desequilibrio en la temperatura corporal relacionada con alteración de la tasa metabólica.

\section{Objetivo}

Mantener una temperatura corporal durante el tratamiento y hasta el cierre de la herida.

\section{Intervenciones}

Proteger a la herida de agresiones externas físicas, químicas y bacterianas.

Minimizar los cambios de apósitos.

Registro de la úlcera en cada curación.

\section{Evaluación}

Se mantiene la temperatura entre 36.5 y $37.5^{\circ} \mathrm{C}$, durante el tratamiento.

Necesidad: Higiene / Piel

\section{Diagnóstico de enfermería 6}

Deterioro de la integridad tisular relacionado con disminución de la circulación, manifestado por lesión por destrucción tisular observándose hueso sacro.

\section{Objetivo}

Recuperar la integridad de los tejidos.

\section{Intervenciones}

Valoración continúa de la lesión durante el procedimiento de curación.

Realizar curaciones, colocando el sistema VAC.

Aplicación de terapia húmeda durante tiempo corto.

Determinar tiempo de cierre quirúrgico.

Cuidados a piel perilesional.

\section{Evaluación}

Resolución de la lesión en 12 semanas de tratamiento.

Necesidad: Seguridad

\section{Diagnóstico de enfermería 8}

Riesgo potencial de dolor agudo relacionado con el procedimiento de curación.

\section{Objetivo}

Controlar el dolor durante el procedimiento de curación.

\section{Intervenciones}

Considerar la necesidad de analgesia antes del procedimiento.

Mantener la terapia en modalidad continua y con intensidad media.

Realizar curaciones dos veces por semana.

\section{Evaluación}

El paciente refiere mínimo dolor durante el procedimiento de curación.

Necesidad: Comunicación

\section{Diagnóstico de enfermería 9}

Deterioro de la interacción social relacionado con la limitación de la movilidad física manifestado por interacción disfuncional con los compañeros, familia o amigos.

\section{Objetivo}

Impulsar la comunicación y las actividades sociales.

\section{Intervenciones}

Hacer que el paciente participe en la toma de decisiones para la aplicación de tratamiento.

Fomentar a participar en actividades sociales.

\section{Evaluación}

El paciente realiza actividades fuera de su domicilio (ir a eventos sociales).

Necesidad: Aprendizaje

\section{Diagnóstico de enfermería 10}

Conocimientos deficientes en relación a su tratamiento relacionado con poca familiaridad con los recursos del tratamiento manifestado por verbalización del problema.

\section{Objetivo}

Conocer el tratamiento de la úlcera por presión.

\section{Intervenciones}

Explicar al paciente el procedimiento a realizar.

Dar a conocer los diferentes tipos de tratamiento, (terapia húmeda, presión negativa, intervención quirúrgica).

Exponer los factores de riesgo para la formación de nuevas úlceras por presión.

Fomentar el autocuidado, mediante su participación continua en actividades cotidianas.

\section{Evaluación}

Es capaz de elegir el tratamiento más adecuado y cómodo para la lesión.

\section{EVOLUCIÓN}

Úlcera por presión en región sacra cubierta con tejido necrótico, se decide eliminar la escara. (Figura 1) 
Al eliminar la placa necrótica se observa úlcera con 95\% de tejido esfacelado, borde necrótico, exudado escaso, eritema perilesional y sin referir dolor a la manipulación. (Figura 2)

Se deja sistema de succión continua y se realizan cambios cada 48 horas, observándose limpieza de la úlcera descubriéndose tejido graso y hueso sacro. (Figura 3 y 4)

Cuatro semanas después de iniciado el tratamiento se observa $70 \%$ de tejido de granulación. La úlcera mide $6.5 x$ $4 \mathrm{~cm}$. tunelización hacia las 12 horas de $18 \mathrm{~cm}$, hacia las 3 de $6 \mathrm{~cm}$. (Figura 5)

Ocho semanas de tratamiento, úlcera en proceso de contracción, 95 \% de tejido de granulación. Sin presencia de tunelizaciones. (Figuras 6 y 7 )

Doce semanas de tratamiento y se observa úlcera con $100 \%$ de tejido de granulación y en contracción, sin embargo se desarrolla sobrecrecimiento de hiperqueratosis formando callo en el borde de la herida por lo que se considera el momento ideal para cierre quirúrgico. (Figura 8)

Se realiza cierre de úlcera con colgajo miocutáneo manteniéndose durante una semana con drenaje subcutáneo y

\section{Figura 1}

Escara necrótica en úlcera sacra

\section{Figura 2}

Úlcera por presión al retirar tejido necrótico
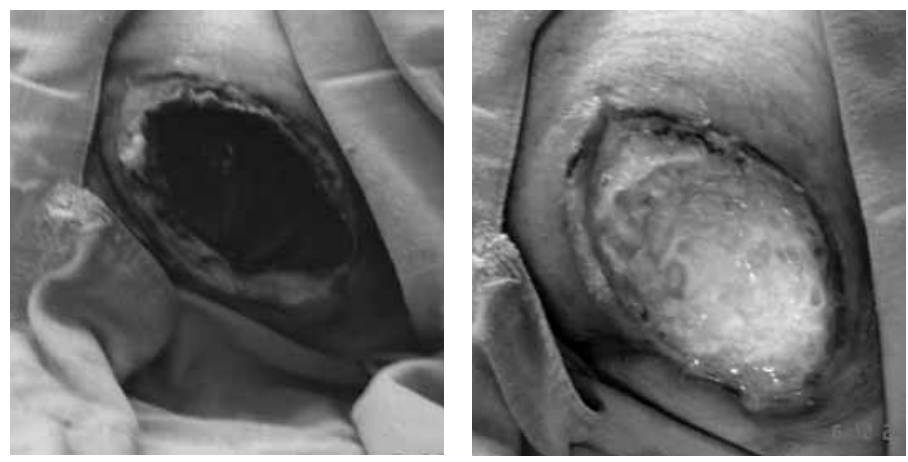

\section{Figura 3 y 4}

Utilización de terapia con presión negativa y evolución a los 4 días
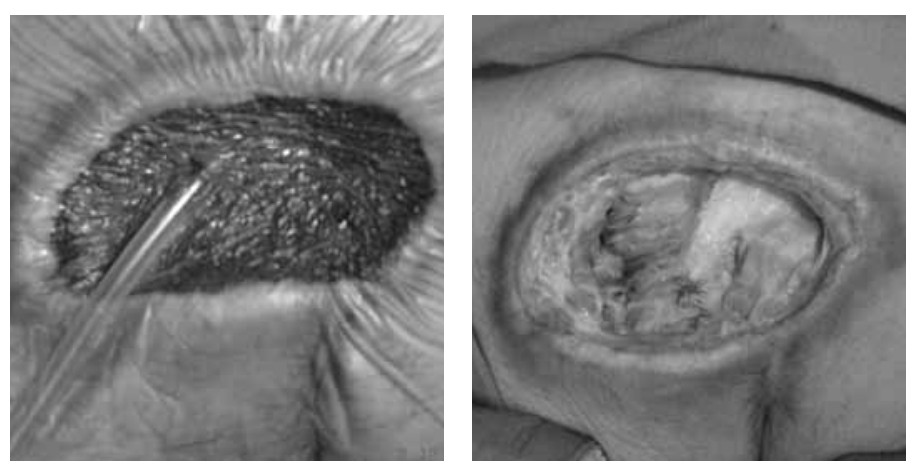

posteriormente se retiran drenajes y suturas obteniéndose excelentes resultados. (Figuras 9 y 10)

\section{RESULTADOS}

El paciente presentó mejoría desde el inicio de tratamiento, a partir del retiro del tejido necrótico no se vuelve a registrar alteración en la temperatura corporal. Se logra egresar del Hospital a los 11 días con seguimiento por medio de la Consulta Externa, realizándose curaciones cada 48 a 72 horas con mínima manipulación. Se utiliza Sistema VAC portátil que proporciona movilidad al paciente y le permite participar en las actividades cotidianas al ser una unidad de terapia ligera y manejable, y al tener una batería interna de larga duración. El paciente permanece con el sistema durante 12 semanas hasta el cierre quirúrgico, que se dispone al encontrarse la herida con $100 \%$ de tejido de granulación, sin presencia de infección, ni tunelizaciones, y el paciente en condiciones clínicas favorables para recibir el colgajo.

\section{Figura 5}

Doce semanas de tratamiento

\section{Figura 6}

8 semanas de tratamiento con sistema VAC
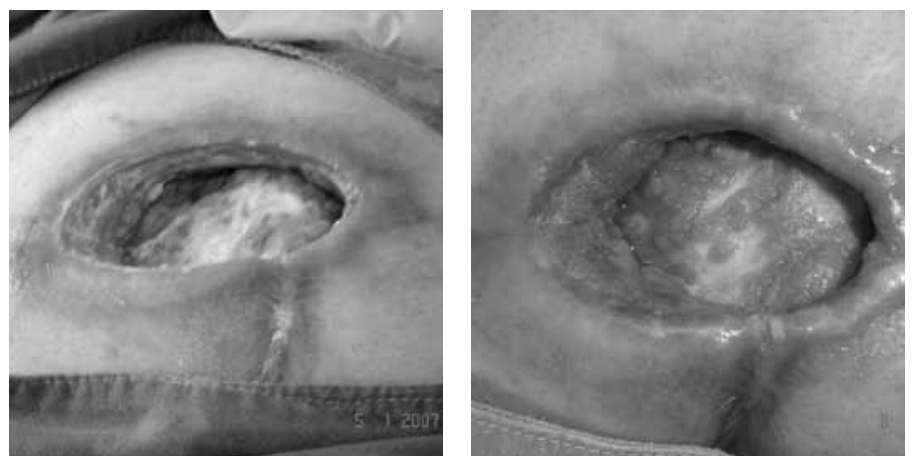

\section{Figura 7}

VAC

\section{Figura 8}

Doce semanas de tratamiento
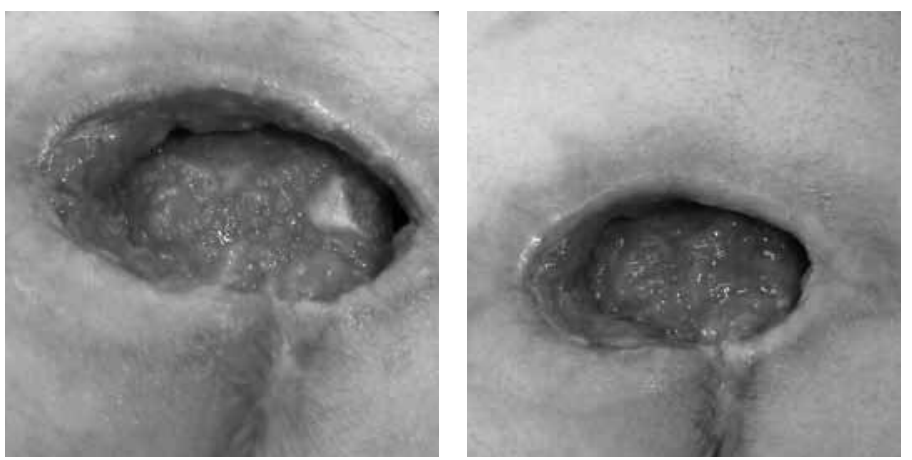
Figura 9 y 10

Cierre quirúrgico

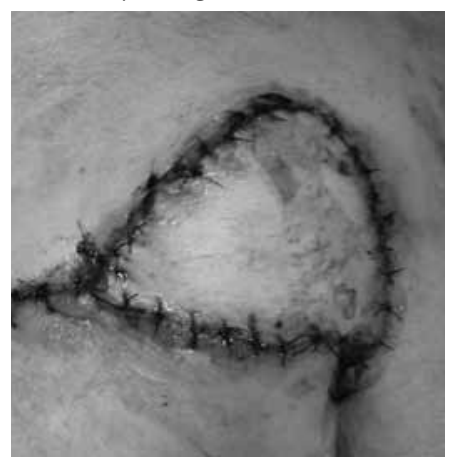

\section{DISCUSIÓN}

El método más eficaz para tratar una úlcera por presión es su prevención, minimizando los factores de riesgo, estableciendo medidas posturales, extremando la limpieza y eliminando las condiciones que favorecen su aparición.

Básicamente el cuidado local de la úlcera incluye limpieza, desbridamiento, prevención y tratamiento de infecciones, así como favorecer la aparición del tejido de granulación; ${ }^{7}$ para asegurar el éxito del cierre ya sea quirúrgico o por segunda intención se requiere preparar el lecho de la herida de manera adecuada eliminando las barreras que impiden la cicatrización como son el tejido necrótico, el desequilibrio microbiano y el exudado excesivo, de esta manera se evitan las complicaciones más frecuentes como son: seroma, hematoma, necrosis cutánea superficial, dehiscencia de la sutura, infección y pérdida del colgajo. De acuerdo a lo reportado la mayoría de las úlceras por presión de $3^{\text {er }}$ y $4^{\circ}$ grado requieren intervención quirúrgica que consiste en la desbridación del tejido necrosado y cicatrizal hasta visualizar el tejido normal incluso con remoción ósea parcial de ser necesario, para el cierre está indicado el uso de colgajos musculares o musculocutáneos para proveer una adecuada cubierta. ${ }^{8}$

El uso de presión negativa para el tratamiento de una úlcera por presión grado IV es efectivo, existen estudios donde se describe la reducción en el tiempo y en el costo, reportando disminución hasta de un $60 \%$ de días de tratamiento comparado con terapia húmeda y un ahorro hasta de 9, 000 dólares por úlcera. ${ }^{9}$

\section{CONCLUSIONES}

Para el tratamiento de esta úlcera por presión en todo momento se contempló al paciente en su totalidad y su entorno, ya que de esto depende la decisión de tratamiento y el éxito del cierre de la úlcera en su totalidad. La terapia asistida por vacío ha mostrado su eficacia permitiendo acelerar el proceso de formación de tejido de granulación y disminuir en forma importante el tamaño de la lesión, logrando prepararlo para recibir colgajo, por otro lado al convertirse en una herida cerrada y controlada evita la presencia de infección, permitió egresar al paciente ya que se utilizó un sistema portátil con lo que se logró que regresara a sus actividades cotidianas.

Debido a que la úlcera se detectó 4 meses después de que se generó no se logro el cierre total con el sistema VAC, sin embargo la detección y tratamiento oportuno de estas úlceras permite que la terapia con VAC logre el cierre total de estas lesiones hasta en 8 semanas.

\section{REFERENCIAS BIBLIOGRAFICAS}

1 García DO. Manual de Cirugía Plástica. Úlceras por presión [en línea] http://www.secpre.org/documentos (consulta en febrero 2007)

2 Ortega-V; María Carolina. Manual de Evaluación del Servicio de Calidad de Enfermería Estrategias para su aplicación. Ed. Medica Panamericana: México; 2006.

3 Carrasco MVH. Salud del Anciano Úlceras por presión. [en línea] www.nacom.es/docs/Salud/web_saludalia/tu_salud/ doc/anciano/doc/doc_úlceras_por_presion.htm (consulta agosto 2004)

4 Andrades P, Sepúlveda S, González J. Curación avanzada de heridas. Revista chilena de cirugía. (56) 4; Junio 2004; Págs. 396-403.

5 Como funciona V.A.C. ${ }^{\circledR}$ Therapy. [en línea]; www.kci-medical. $\mathrm{com} / \mathrm{kci} / \mathrm{spain} /$ terapiaskci/vactherapy/comofuncionavactherapy.htm (consulta mayo 2007)

6 Canet-Bolado;C. Lamalfa-Díaz;E. Mata-Morante;M. Cols. Manual de prevención y tratamiento de úlceras por presión. Hospital Universitario, Dirección de Enfermería, Área de Formación y Calidad: España; 2003.

7 Delgado-Fernández; R. Pérez-Vázquez; Angeles. RodríguezIglesias; J. Cols. Manual de prevención y tratamiento de úlceras por presión. Xunta de Galicia, División de Asistencia Sanitaria: Santiago de Compostela España; 2005.

8 Clínicas del Hospital General de México, O. D. Cirugía Plástica. Piensa: México; 2003.

9 Philbeck TE. Eficacia clínica y rentabilidad del tratamiento de heridas con presión negativa aplicada externamente en pacientes de Medicare atendidos en su domicilio [en línea] www.kci-medical.com/kci/spain/evidenciasclínicas/ vac2.2.htm (consulta enero 2007)

\section{DIRECCIÓN PARA CORRESPONDENCIA}

Lic. Imelda Flores Montes: imeldafm@hotmail.com 